\title{
Compatibility of Biosimilar Filgrastim with Cytotoxic Chemotherapy during the Treatment of Malignant Diseases (VENICE): A Prospective, Multicenter, Non-Interventional, Longitudinal Study
}

Stefan Fruehauf · Burkhard Otremba · Oliver Stötzer •

Christine Rudolph

Received: August 10, 2016 / Published online: October 14, 2016

(C) The Author(s) 2016. This article is published with open access at Springerlink.com

\section{ABSTRACT}

Introduction: Febrile neutropenia (FN) is a serious and frequent complication of cytotoxic chemotherapy. Biosimilar filgrastim (Nivestim $^{\mathrm{TM}}$, Hospira Inc, A Pfizer Company, Lake Forest, IL, USA) is a granulocyte-colony stimulating factor licensed for the treatment of neutropenia and FN induced by myelosuppressive chemotherapy. The primary goal of this VENICE study (ClinicalTrials.gov identifier, NCT01627990) was to observe the tolerability, safety and efficacy of biosimilar

Enhanced content To view enhanced content for this article go to http://www.medengine.com/Redeem/ CAF6F0602B26798D.

S. Fruehauf $(\bowtie)$

Klinik Dr. Hancken, Hämatologie, Onkologie und Palliativmedizin, Stade, Germany

e-mail: stefan_fruehauf@urz.uni-heidelberg.de

B. Otremba

Onkologische Praxis Oldenburg/Delmenhorst,

Standort Oldenburg, Oldenburg, Germany

O. Stötzer

Hämato-Onkologische Schwerpunktpraxis, Praxis

München, Munich, Germany

C. Rudolph

Hospira Deutschland GmbH, A Pfizer Company, Medical Affairs, Munich, Germany filgrastim in patients receiving cancer chemotherapy.

Methods: This was a prospective, multicenter, non-interventional, longitudinal study. Consenting adult patients with solid tumors or hematologic malignancies for whom cytotoxic chemotherapy and treatment with biosimilar filgrastim was planned were enrolled.

Results: Among the enrolled patients $(N=386)$, $81 \%$ were female, with a median age (range) of 61 (22-92) years, with 39\% >65 years old. Most patients $(n=338 ; 88 \%)$ had solid tumors and the remainder $(n=49 ; 13 \%)$ had hematological malignancies. The majority of the patients (64\%) received biosimilar filgrastim as primary prophylaxis and $36 \%$ as secondary prophylaxis. At the follow-up visits, for the majority of patients $(95.6 \%)$ there had been no change in chemotherapy dose due to FN. For two patients $(0.5 \%)$ the chemotherapy was discontinued due to $\mathrm{FN}$ and for four patients (1.0\%) the chemotherapy dose was reduced due to FN. For the majority of patients (96.9\%) the chemotherapy cycle following the first biosimilar filgrastim treatment was not delayed due to FN. For 3 patients $(0.8 \%)$, the chemotherapy was delayed following the first 
biosimilar filgrastim treatment. Less than one-third $(29.8 \%)$ of the patients experienced $\geq 1$ adverse event that was at least potentially related to biosimilar filgrastim treatment.

Conclusions: Biosimilar filgrastim was effective and well-tolerated in both the primary and secondary prophylactic setting in patients undergoing chemotherapy for solid tumors and hematological malignancies.

Trial Registration: ClinicalTrials.gov identifier, NCT01627990.

Funding: Hospira Inc, A Pfizer Company, Lake Forest, IL, USA.

Keywords: Biosimilar; Chemotherapy; Filgrastim; Granulocyte-colony stimulating factor; Hematology; Neutropenia; Nivestim; Oncology

\section{INTRODUCTION}

Chemotherapy-induced neutropenia (CIN) is a common and serious complication of myelosuppressive chemotherapy [1]. Neutropenia is associated with life-threatening infections and may delay the chemotherapy schedule, having a negative impact on early and long-term outcomes [2]. It is associated with significant morbidity and mortality, and may increase the overall cost of providing cancer therapy [3]. The results of three large-scale studies demonstrated that primary prophylaxis with a granulocyte-colony stimulating factor (G-CSF) significantly reduces the incidence of febrile neutropenia (FN) resulting from cytotoxic chemotherapy [4-6]. Thus, G-CSF products reduce the risk of infectious complications and with it, the consequent loss of therapeutic options, which may result from neutropenia [7]. The use of a G-CSF plus antibiotics may reduce hospitalization time and improve the ability to achieve neutrophil recovery in individuals with chemotherapy-induced FN [8].

Several G-CSF biosimilar filgrastim products [Ratiograstim $^{\circledR}$ (Ratiopharm, Ulm, Germany) Tevagrastim $^{\circledR}$ (Teva, Petah Tikva, Israel), Biograstim $^{\circledR}$ (CT Arzneimittel, Ulm, Germany) Zarzio $^{\circledR}$ (Sandoz, Basel, Switzerland) Nivestim ${ }^{\circledR}$ (Hospira Inc, A Pfizer Company, Lake Forest, IL, USA)] [9] to the reference product Neupogen ${ }^{\circledR}$ (Amgen, Thousand Oaks, USA) have been approved in Europe [10]. All these products are manufactured in facilities with state-of-the-art technology and have passed the regulatory requirements for approval, mainly phase I and phase III trials, with the consequent pharmacodynamic/pharmacokinetic (PD/PK) evaluations and studies on efficacy and safety; however, there are still some unresolved questions regarding their long-term evaluation, in particular, the limited real-world experience at the time of approval of these products in terms of efficacy, safety and immunogenicity.

Biosimilar filgrastim (Nivestim) is a 175-amino acid protein recombinant methionyl human G-CSF, with a molecular weight of $18,800 \mathrm{Da}$, that is produced in Escherichia coli [11]. The active substance is non-glycosylated protein, containing an $\mathrm{N}$-terminal methionyl extension. It stimulates the proliferation, differentiation and activation of late progenitor cells of the granulocyte lineage and enhances the activity of mature neutrophils.

Since the use of biosimilar filgrastim has not yet been sufficiently documented in the context of day-to-day medical practice, the goal of the VENICE study, (i.e., Compatibility of Nivestim with Cytotoxic Chemotherapy in the Treatment of Malignant Diseases) (ClinicalTrials.gov identifier, NCT01627990) was to assess the tolerability, safety and efficacy of prophylactic 
biosimilar filgrastim to reduce the duration of neutropenia and the incidence of $\mathrm{FN}$ in patients receiving cytotoxic cancer chemotherapy [12]. The VENICE study was a multicenter, prospective, longitudinal, observational study designed to evaluate the use of biosimilar filgrastim in 'real-world' clinical practice.

\section{METHODS}

All procedures followed were in accordance with the ethical standards of the responsible committee on human experimentation (institutional and national) and with the Helsinki Declaration of 1964, as revised in 2013. Informed consent was not required from patients as this was a non-interventional observational trial.

\section{Patient Population}

The study enrolled children and adults of either gender with solid or malignant hematological tumors who were scheduled to undergo prophylactic treatment with biosimilar filgrastim (Nivestim) to shorten the duration of a neutropenia, or to prevent the occurrence of chemotherapy-induced FN. Reasons for exclusion from the study included chronic myeloid leukemia or myelodysplastic syndrome, hypersensitivity to any component of the biosimilar filgrastim product, not undergoing chemotherapy, or being treated curatively with G-CSF.

\section{Primary Outcome Measures}

The primary objective was to assess the tolerability, safety and efficacy of prophylactic treatment using biosimilar filgrastim in patients receiving cytotoxic chemotherapy for cancer, with an emphasis on patients undergoing in-patient treatment.

\section{Secondary Outcome Measures}

The secondary objectives were to describe the characteristics of patients being treated with biosimilar filgrastim; the treatment modalities using biosimilar filgrastim; and the characteristics of participating physicians and their prescribing practices with regard to G-CSF.

\section{Subgroups}

Patients were stratified according to age (18-65 years old and $>65$ years old) and tumor type (hematological vs. solid tumors). Other parameters used for stratification analysis included treatment site, infections (type, affected area and causative organism), route of administration (subcutaneous vs. intravenous) and adverse events (AEs) (seriousness, severity, and outcome).

\section{Data Collection and Statistical Analysis}

Patient data were gathered during the initial enrollment visit and two subsequent follow-up visits. AEs occurring during the study were recorded regardless of causality. The intent-to-treat (ITT) population consisted of all patients enrolled in the study. The safety (SAF) population included all patients who had at least one follow-up visit or a documented $\mathrm{AE}$. The efficacy analysis set (probability proportionate to size [PPS]) included patients from the safety analysis set who fulfilled all the inclusion/exclusion criteria and whose baseline absolute neutrophil count (ANC) and one other ANC value had been documented during therapy or shortly afterwards. 
Reported data were analyzed descriptively. Descriptive $p$ values and confidence intervals were calculated for the parameter of major interest. For hospitalizations due to $\mathrm{FN}$ or infections, all incidents occurring outside the study duration were disregarded. Multiple documentations of patient hospitalizations occurring on the same date were regarded as one single hospitalization. The incidence of hospitalizations due to $\mathrm{FN}$ or infections are presented as the absolute number of events, the event rate per patient, the event rate per patient-years, the absolute incidence, the incidence rate per patient and the incidence rate per patient-years. As each patient could have been hospitalized more than once, a multivariate logistic regression model was used to investigate the influence of selected risk factors on hospitalization. The risk factors of interest were age ( $\leq 65$ years of age, $>65$ years of age), tumor type (hematological or solid tumor), and number of prior chemotherapy regimens $(\leq 1, \geq 2)$.

\section{Adverse Events}

AEs occurring before the screening visit were disregarded. Other AEs were coded according to Medical Dictionary for Regulatory Activities (MedDRA) System Organ Class (SOC) and the Preferred Term (PT). These were tabulated in terms of numbers of patients with $\geq 1 \mathrm{AE}$, percent with respect to the analyzed population and the number of AEs. The duration of AEs was analyzed using summary statistics.

\section{Sample Size Estimation}

A precision of \pm 3 percent was planned, assuming a probability of $20 \%$ for the occurrence of hospitalization due to $\mathrm{FN}$ or infection for each patient recruited during the observation period. Assuming a confidence level of $5 \%$, a sample of 700 eligible patients was planned to fulfill requirements for power.

\section{RESULTS}

Forty-eight study sites participated in the study, each contributing 2-60 patients per site. The ITT population included 386 adult patients undergoing cytotoxic chemotherapy who were prescribed biosimilar filgrastim to reduce the duration of neutropenia and to reduce the incidence of chemotherapy-induced FN. The SAF population included 382 patients (99.0\%) and the PPS population included 185 patients from the SAF (48.4\%). Data for most patients (84.4\%) was available for all three study visits and the median duration of observation was 64 days.

\section{Demographic and Baseline Characteristics}

Most study participants (81\%) were female, with a median age (range) of 61 (22-92) years and $39 \%$ of the patients age were $>65$ years old, having solid tumors $(n=338,88 \%)$ or hematological malignancies $(n=49,13 \%)$. One patient had both a solid tumor and a hematological malignancy, but was counted in the solid tumor group. Other demographic and baseline clinical characteristics are summarized in Table 1.

\section{Medical History}

At baseline, $46.1 \%$ of patients reported at least 1 prior diagnosis, $30.3 \%$ reported at least 2 prior diagnoses and $18.4 \%$ reported at least 3 other prior diagnoses. The number of prior diagnoses was similar for all tumor types, with the most frequently reported cardiovascular disease 
Table 1 Demographic and baseline characteristics

\begin{tabular}{|c|c|c|c|c|}
\hline Parameter & $\begin{array}{l}\text { Hematological } \\
\text { malignancies }(N=48)\end{array}$ & $\begin{array}{l}\text { Breast cancer } \\
(N=233)\end{array}$ & $\begin{array}{l}\text { Other solid } \\
\text { tumors }(N=105)\end{array}$ & $\begin{array}{l}\text { Total } \\
(N=386)\end{array}$ \\
\hline \multicolumn{5}{|l|}{ Gender, $n(\%)$} \\
\hline Male & $24(50.0)$ & $1(0.4)$ & $50(47.6)$ & $75(19.4)$ \\
\hline Female & $24(50.0)$ & $232(99.6)$ & $55(52.4)$ & $311(80.6)$ \\
\hline $\begin{array}{l}\text { Median age }(\min , \max ), \\
\text { years }\end{array}$ & $68.0(22.0,80.0)$ & $58.0(23.0,82.0)$ & $68.0(40.0,92.0)$ & $61.0(22.0,92.0)$ \\
\hline \multicolumn{5}{|l|}{ Age categories, $n(\%)$} \\
\hline $18-65$ & $19(39.6)$ & $173(74.2)$ & $45(42.9)$ & $237(61.4)$ \\
\hline$>65$ & $29(60.4)$ & $60(25.8)$ & $60(57.1)$ & $149(38.6)$ \\
\hline \multirow{2}{*}{$\begin{array}{l}\text { Median height (min, } \max ) \text {, } \\
\mathrm{cm}(n)\end{array}$} & 48 & 232 & 102 & 382 \\
\hline & $171.0(152.0,192.0)$ & $165.0(150.0,186.0)$ & $169.5(140.0,194.0)$ & $166.0(140.0,194.0)$ \\
\hline \multirow{2}{*}{$\begin{array}{l}\text { Median body weight } \\
(\min , \max ), \operatorname{kg}(n)\end{array}$} & 45 & 229 & 102 & 376 \\
\hline & $70.0(41.0,123.0)$ & $71.0(45.0,119.0)$ & $71.0(43.0,159.0)$ & $71.0(41.0,159.0)$ \\
\hline \multirow{2}{*}{$\begin{array}{l}\text { Median systolic BP } \\
\quad(\min , \max ), \operatorname{mmHg}(n)\end{array}$} & 27 & 176 & 67 & 270 \\
\hline & $120.0(100.0,160.0)$ & $130.0(99.0,176.0)$ & $129.0(105.0,170.0)$ & $130.0(99.0,176.0)$ \\
\hline \multirow{2}{*}{$\begin{array}{l}\text { Median diastolic BP } \\
\quad(\min , \max ), \operatorname{mmHg}(n)\end{array}$} & 27 & 175 & 67 & 269 \\
\hline & $80.0(50.0,90.0)$ & $80.0(50.0,115.0)$ & $75.0(60.0,99.0)$ & $80.0(50.0,115.0)$ \\
\hline \multirow{2}{*}{$\begin{array}{l}\text { Median body temperature } \\
\quad(\min , \max ),{ }^{\circ} \mathrm{C}(n)\end{array}$} & 14 & 95 & 47 & 156 \\
\hline & $36.2(35.2,37.7)$ & $36.5(35.5,38.5)$ & $36.5(35.0,37.3)$ & $36.5(35.0,38.5)$ \\
\hline
\end{tabular}

Patient 054-002 had a solid tumor as well as a hematological malignancy but was counted in the stratified analyses under solid tumors

$B P$ blood pressure, CD34 hematopoietic progenitor cell antigen CD34

(26.9\%), chronic obstructive pulmonary disease, renal and liver failure reported (for each, $<5 \%)$, FN (2.6\%), recurrent infections (3.9\%) and human immunodeficiency virus (HIV) infection (0.5\%) (Table 2).

Approximately half of enrolled patients (42.5\%) had received prior chemotherapy (Table 3), but only $23.3 \%$ had received a single prior regimen. Among the patients for whom this information was available $(n=158), 4.4 \%$ reported an episode of $\mathrm{FN}$ after the most recent chemotherapy and
$36.5 \%$ of the patients had no FN history after chemotherapy. Overall, $19.4 \%$ of patients had undergone prior radiotherapy, with a higher rate among patients with other tumors (33.3\%).

Although hypotension, dermatitis, mucositis, sepsis and erysipelas are risk factors associated with FN, very few patients reported these conditions at baseline. The most frequent was mucositis (12.7\%), followed by hypotension (1.8\%), dermatitis $(0.8 \%)$ and erysipelas $(0.3 \%)$. 
Table 2 Medical history by tumor type (all-patient set)

\begin{tabular}{|c|c|c|c|c|}
\hline Previous medical diagnoses $^{a}$ & $\begin{array}{l}\text { Hematological } \\
\text { malignancies }(N=48)\end{array}$ & $\begin{array}{l}\text { Breast cancer } \\
(N=233)\end{array}$ & $\begin{array}{l}\text { Other solid } \\
\text { tumors }(N=105)\end{array}$ & $\begin{array}{l}\text { Total } \\
(N=386)\end{array}$ \\
\hline Febrile neutropenia, $n$ (\%) & $2(4.2)$ & $6(2.6)$ & $2(1.9)$ & $10(2.6)$ \\
\hline Recurrent infections, $n(\%)$ & $3(6.3)$ & $8(3.4)$ & $4(3.8)$ & $15(3.9)$ \\
\hline HIV infection, $n(\%)$ & $1(2.1)$ & $1(0.4)$ & 0 & $2(0.5)$ \\
\hline COPD, $n(\%)$ & $2(4.2)$ & $3(1.3)$ & $10(9.5)$ & $15(3.9)$ \\
\hline Cardiovascular diseases, $n(\%)$ & $12(25.0)$ & $54(23.2)$ & $38(36.2)$ & $104(26.9)$ \\
\hline Renal failure, $n(\%)$ & $2(4.2)$ & $2(0.9)$ & $5(4.8)$ & $9(2.3)$ \\
\hline Liver failure, $n(\%)$ & 0 & $2(0.9)$ & 0 & $2(0.5)$ \\
\hline Other or at least one other diagnosis, $n$ (\%) & $24(50.0)$ & $97(41.6)$ & $57(54.3)$ & $178(46.1)$ \\
\hline At least two other diagnoses, $n(\%)$ & $14(29.2)$ & $63(27.0)$ & $40(38.1)$ & $117(30.3)$ \\
\hline At least three other diagnoses, $n(\%)$ & $7(14.6)$ & $42(18.0)$ & $22(21.0)$ & $71(18.4)$ \\
\hline
\end{tabular}

\section{Tumor Entities}

Forty-nine patients (12.7\%) had a hematological malignancy, including Hodgkin's lymphoma $\quad(n=5, \quad 1.3 \%)$, non-Hodgkin's lymphoma $(n=22 ; 5.7 \%)$, monoclonal gammopathy (multiple myeloma or Kahler disease; $n=9,2.3 \%$ ), chronic lymphocytic leukemia $(n=9,2.3 \%)$ or other hematological malignancies $(n=4,1.0 \%)$. The median duration of disease at baseline was 3.0 years (range $1.0-18.0$ years). Only three patients $(6.1 \%)$ had undergone autologous stem cell transplantation. The disease classification used for the hematological malignancies was not consistent across patients; however, the results indicate that patients were distributed from early to late staging groups. Most patients (87.6\%) had solid tumors and their median duration of disease at baseline was 2.0 years (range, 1.0-52.0 years). Breast cancer was the largest group among solid tumor patients (68.9\%). Other tumor types are summarized in Table 4. Based on the TNM classification system, the majority of solid tumors were small, low-grade cancers with no metastasis, although they ranged from Stage I to Stage IV.

\section{Chemotherapy}

The goal of chemotherapy for all patients at enrollment was adjuvant (49\%) or curative (31.9\%) (Table 5); with treatment being predominantly curative among patients with hematological malignancies (64.6\%), but predominantly adjuvant among patients with breast cancer (63.1\%). Among those with other solid tumors, the goal was adjuvant $(31.4 \%)$ or curative therapy (29.5\%).

For most patients, the planned chemotherapy was first line treatment (63.5\%) and was similar across the tumor subgroups 
Table 3 Chemotherapy history (all-patients set)

\begin{tabular}{llllll}
\hline & & $\begin{array}{l}\text { Hematological } \\
\text { malignancies } \\
(\boldsymbol{N = 4 8 )}\end{array}$ & $\begin{array}{l}\text { Breast cancer } \\
(\boldsymbol{N = 2 3 3 )}\end{array}$ & $\begin{array}{l}\text { Other solid } \\
\text { tumors }(\boldsymbol{N}=\mathbf{1 0 5})\end{array}$ & $\begin{array}{l}\text { Total } \\
(\boldsymbol{N}=\mathbf{3 8 6})\end{array}$ \\
\hline $\begin{array}{l}\text { Prior chemotherapy, } n \text { (\%) } \\
\text { Number of prior chemotherapy } \\
\text { regimens, } n \text { (\%) }\end{array}$ & 0 & $32(66.7)$ & $70(30.0)$ & $62(59.0)$ & $164(42.5)$ \\
& 1 & $14(29.2)$ & $43(18.5)$ & $33(31.4)$ & $6(1.6)$ \\
& 2 & $4(8.3)$ & $6(2.6)$ & $12(11.4)$ & $22(5.7)$ \\
& 3 & $4(8.3)$ & $7(3.0)$ & $8(7.6)$ & $19(4.9)$ \\
& 4 & $2(4.2)$ & $3(1.3)$ & $1(1.0)$ & $6(1.6)$ \\
& 5 & $1(2.1)$ & $1(0.4)$ & $1(1.0)$ & $3(0.8)$ \\
& 6 & 0 & $3(1.3)$ & $2(1.9)$ & $5(1.3)$ \\
& 8 & $1(2.1)$ & $2(0.9)$ & 0 & $3(0.8)$ \\
& $1(2.1)$ & $1(0.4)$ & 0 & $2(0.5)$ \\
& 60 & 0 & 0 & $1(1.0)$ & $1(0.3)$ \\
& 18 & 165 & 46 & 229 \\
\hline
\end{tabular}

(Table 5). For most, the treatment cycle duration was 21 days (66.8\%) and was planned for six cycles $(46.9 \%)$. At enrollment, most patients were beginning their chemotherapy $(31.6 \%)$ or had already received one cycle (29.8\%). Most patients (65.3\%) did not have G-CSF prescribed during the first cycle (Table 6).

The most common chemotherapeutic agents received by patients with hematological malignancies were cyclophosphamide (66.7\%), rituximab (58.3\%), doxorubicin (39.6\%) and vincristine (39.6\%) (Table 7). Patients with breast cancer most frequently received cyclophosphamide $\quad(72.5 \%), \quad$ epirubicin (59.7\%), docetaxel (37.8\%), 5-fluorouracil (30.5\%) and paclitaxel (26.6\%). Patients with other solid tumors most frequently received carboplatin (35.2\%), 5-fluorouracil (24.8\%) and paclitaxel (21.0\%).

Most patients with solid tumors $(88.0 \%$ of breast cancer, $88.6 \%$ of other solid tumors) did not receive antibiotic prophylaxis; however,
$58.3 \%$ of patients with hematological malignancies were prophylactically treated with antibiotics. Most (96.6\%) did not receive adjuvant radiotherapy.

During follow-up visits, for the majority of patients (95.6\%) there had been no change in chemotherapy dose due to FN. For two patients $(0.5 \%)$ the chemotherapy was discontinued due to $\mathrm{FN}$ and for four patients (1.0\%) the chemotherapy dose was reduced due to FN. For the majority of patients (96.9\%) the chemotherapy cycle following the first biosimilar filgrastim treatment was not delayed due to FN. For three patients (0.8\%), the chemotherapy was delayed following the first biosimilar filgrastim treatment.

Initial antibiotic prophylaxis was administered to $12.7 \%$ of patients with hematologic malignancies $(n=20,41.7 \%)$ and solid tumors $(n=29,8.6 \%)$. Most did not receive initial antibiotic prophylaxis. 
Table 4 Solid tumor types (all-patients set), $N=338$

\begin{tabular}{|c|c|}
\hline Location & \\
\hline \multicolumn{2}{|l|}{ Lung, $n(\%)$} \\
\hline Small cell lung cancer & $19(5.6)$ \\
\hline Non-small cell lung cancer & $9(2.7)$ \\
\hline \multicolumn{2}{|l|}{ Head/neck, $n$ (\%) } \\
\hline Oral & $1(0.3)$ \\
\hline Esophagus $^{\mathrm{a}}$ & $1(0.3)$ \\
\hline \multicolumn{2}{|l|}{ Gynecological, $n$ (\%) } \\
\hline Breast & $233(68.9)$ \\
\hline Ovaries & $21(6.2)$ \\
\hline Endometrium & $3(0.9)$ \\
\hline Cervical & $4(1.2)$ \\
\hline \multicolumn{2}{|l|}{ Urological, $n(\%)$} \\
\hline Bladder & $3(0.9)$ \\
\hline Testicle & $1(0.3)$ \\
\hline Kidney & $1(0.3)$ \\
\hline Prostate & $5(1.5)$ \\
\hline \multicolumn{2}{|l|}{ Digestive system, $n(\%)$} \\
\hline Colon/rectum & $15(4.4)$ \\
\hline Stomach & $6(1.8)$ \\
\hline Pancreas & $9(2.7)$ \\
\hline Esophagus $^{\mathrm{a}}$ & $1(0.3)$ \\
\hline Other, $n(\%)$ & $7(2.1)$ \\
\hline
\end{tabular}

${ }^{a}$ Two cases of esophageal cancer were placed in different body locations

Only seven patients (1.8\%) had chemotherapy that was accompanied by radiotherapy.

\section{Weight, Vital Signs, Hospitalizations}

Little change was observed in the median weight and vital signs across tumor types during the course of the study. No changes in weight or vital signs were observed when evaluated by age subgroup. Twenty-four patients had $\geq 1$ hospitalizations due to general physical health deterioration $(n=6)$, back pain, spinal pain, device-related infection, diarrhea, leukopenia, spinal pain and vomiting (for each, $n=2$ ). The median duration of hospitalization for patients with hematological malignancies was 8 days ( $n=11$; range $5-40$ days) vs. 16.5 days $(n=38$, range 2-33 days) for patients with solid tumors. Two hospitalizations due to FN or infections were reported for patients with hematological malignancies vs. seven for patients with solid tumors.

The hospitalization rate for FN or infections was $4.3 \%$ for the hematological malignancy group and $2.1 \%$ for the solid tumor group. The number of hospitalizations due to $\mathrm{FN}$ or infections per patient-year was 0.2 for the hematological malignancy group, 0.1 for the breast cancer group and 0.1 for the other solid tumor group. When number of hospitalizations due to FN or infections was stratified by age, patients $\leq 65$ years old had more hospitalizations (seven vs. two) and a higher rate of hospitalizations (3.0 vs. 1.4 per 100 patients). In the $\leq 65$-year-old group, $2.5 \%$ were hospitalized during the study vs. $1.4 \%$ in the $>65$-year-old group. Logistic regression confirmed that older patients had a lower risk of hospitalization due to FN or infection [odds ratio (OR) 0.56, 95\% confidence interval (CI) 0.11-2.85]; however, this difference was not statistically significant. Similarly, the risk of hospitalization due to FN or infection was not associated with the number of prior cycles of chemotherapy at baseline (OR 1.07, 95\% CI 0.25-4.55) or tumor type (OR 0.85, 95\% CI 0.10-7.29).

\section{Hematologic Laboratory Values}

Median hemoglobin and thrombocyte values in all tumor subgroups remained relatively 
stable throughout the study from enrollment to last chemotherapy cycle, while median leukocyte values showed a slight increase. For patients with hematological malignancies, the median leukocyte value at first visit was
$3.2 \times 10^{9} / \mathrm{L}$, increasing to $4.1 \times 10^{9} / \mathrm{L}$ before the last chemotherapy cycle (Table 8 ). For patients with breast cancer, the median leukocyte value was $4.3 \times 10^{9} / \mathrm{L}$, increasing to $5.3 \times 10^{9} / \mathrm{L}$, while the median leukocyte value

Table 5 Description of prior chemotherapy, all-patients set

\begin{tabular}{|c|c|c|c|c|c|}
\hline $\begin{array}{l}\text { Chemotherapy } \\
\text { at enrollment }\end{array}$ & & $\begin{array}{l}\text { Hematological } \\
\text { malignancies } \\
(N=48)\end{array}$ & $\begin{array}{l}\text { Breast cancer } \\
(N=233)\end{array}$ & $\begin{array}{l}\text { Other solid } \\
\text { tumors }(N=105)\end{array}$ & $\begin{array}{l}\text { Total } \\
(N=386)\end{array}$ \\
\hline \multirow[t]{2}{*}{ Goal of chemotherapy } & Adjuvant & $9(18.8)$ & $147(63.1)$ & $33(31.4)$ & $189(49.0)$ \\
\hline & Curative & $31(64.6)$ & $61(26.2)$ & $31(29.5)$ & $123(31.9)$ \\
\hline \multirow[t]{4}{*}{ Line of treatment } & 1 & $30(62.5)$ & $151(64.8)$ & $64(61.0)$ & $245(63.5)$ \\
\hline & 2 & $5(10.4)$ & $8(3.4)$ & $23(21.9)$ & $36(9.3)$ \\
\hline & 3 & $4(8.3)$ & $2(0.9)$ & $7(6.7)$ & $13(3.4)$ \\
\hline & $4+$ & $6(12.5)$ & $7(3.0)$ & $4(3.8)$ & $17(4.4)$ \\
\hline \multirow[t]{4}{*}{ Duration of cycles } & 14 Days & $5(10.4)$ & $15(6.4)$ & $17(16.2)$ & $37(9.6)$ \\
\hline & 21 Days & $24(50.0)$ & $175(75.1)$ & $59(56.2)$ & $258(66.8)$ \\
\hline & 28 Days & $12(25.0)$ & $12(5.2)$ & $15(14.3)$ & $39(10.1)$ \\
\hline & Other & $3(6.3)$ & $27(11.6)$ & $11(10.5)$ & $41(10.6)$ \\
\hline \multirow{16}{*}{$\begin{array}{l}\text { Number of originally } \\
\text { planned cycles }\end{array}$} & 0 & 0 & $1(0.4)$ & 0 & $1(0.3)$ \\
\hline & 1 & $2(4.2)$ & 0 & $1(1.0)$ & $3(0.8)$ \\
\hline & 2 & $2(4.2)$ & $1(0.4)$ & $3(2.9)$ & $6(1.6)$ \\
\hline & 3 & $2(4.2)$ & $13(5.6)$ & $6(5.7)$ & $21(5.4)$ \\
\hline & 4 & $4(8.3)$ & $42(18.0)$ & $17(16.2)$ & $63(16.3)$ \\
\hline & 5 & $1(2.1)$ & 0 & $3(2.9)$ & $4(1.0)$ \\
\hline & 6 & $22(45.8)$ & $112(48.1)$ & $47(44.8)$ & $181(46.9)$ \\
\hline & 7 & 0 & $2(0.9)$ & 0 & $2(0.5)$ \\
\hline & 8 & $6(12.5)$ & $14(6.0)$ & 0 & $20(5.2)$ \\
\hline & 9 & $1(2.1)$ & $2(0.9)$ & 0 & $3(0.8)$ \\
\hline & 10 & 0 & $1(0.4)$ & 0 & $1(0.3)$ \\
\hline & 12 & 0 & $3(1.3)$ & $4(3.8)$ & $7(1.8)$ \\
\hline & 13 & 0 & 0 & $1(1.0)$ & $1(0.3)$ \\
\hline & 16 & 0 & $14(6.0)$ & 0 & $14(3.6)$ \\
\hline & 17 & 0 & $1(0.4)$ & $0(0.0)$ & $1(0.3)$ \\
\hline & 18 & 0 & $15(6.4)$ & $1(1.0)$ & $16(4.1)$ \\
\hline
\end{tabular}


Table 5 continued

\begin{tabular}{|c|c|c|c|c|c|}
\hline $\begin{array}{l}\text { Chemotherapy } \\
\text { at enrollment }\end{array}$ & & $\begin{array}{l}\text { Hematological } \\
\text { malignancies } \\
(N=48)\end{array}$ & $\begin{array}{l}\text { Breast cancer } \\
(N=233)\end{array}$ & $\begin{array}{l}\text { Other solid } \\
\text { tumors }(N=105)\end{array}$ & $\begin{array}{l}\text { Total } \\
(N=386)\end{array}$ \\
\hline \multirow[t]{6}{*}{ Number of completed cycles } & 0 & $9(18.8)$ & $85(36.5)$ & $28(26.7)$ & $122(31.6)$ \\
\hline & 1 & $18(37.5)$ & $59(25.3)$ & $38(36.2)$ & $115(29.8)$ \\
\hline & 2 & $6(12.5)$ & $22(9.4)$ & $12(11.4)$ & $40(10.4)$ \\
\hline & 3 & $3(6.3)$ & $33(14.2)$ & $12(11.4)$ & $48(12.4)$ \\
\hline & 4 & $7(14.6)$ & $24(10.3)$ & $4(3.8)$ & $35(9.1)$ \\
\hline & 5 & $2(4.2)$ & $4(1.7)$ & 0 & $6(1.6)$ \\
\hline
\end{tabular}

a Prior treatment was not obtained in some cases

Table 6 Indication for Nivestim, stratified by type of tumor (safety-analysis set)

\begin{tabular}{lllll}
\hline Indication & $\begin{array}{l}\text { Hematological } \\
\text { malignancies }(\boldsymbol{N}=\mathbf{4 7})\end{array}$ & $\begin{array}{l}\text { Breast cancer } \\
(\boldsymbol{N}=\mathbf{2 3 3})\end{array}$ & $\begin{array}{l}\text { Other solid } \\
\text { tumors }(\boldsymbol{N}=\mathbf{1 0 2})\end{array}$ & $\begin{array}{l}\text { Total } \\
(\boldsymbol{N}=\mathbf{3 8 2})\end{array}$ \\
\hline Primary prophylaxis & $22(46.8)$ & $160(68.7)$ & $61(59.8)$ & $243(63.6)$ \\
Secondary prophylaxis & $25(53.2)$ & $73(31.3)$ & $40(39.2)$ & $138(36.1)$ \\
Not recorded & 0 & 0 & 1 & 1 \\
\hline
\end{tabular}

for other solid tumors increased from $2.9 \times 10^{9} /$ L to $5.1 \times 10^{9} /$ L. Similarly, median neutrophil values increased from baseline to last chemotherapy cycle. For patients with hematological malignancies, the median neutrophil value increased from $1.4 \times 10^{9} / \mathrm{L}$ to $2.6 \times 10^{9} /$ L. For patients with breast cancer, the median neutrophil value of $1.6 \times 10^{9} / \mathrm{L}$ increased to $3.1 \times 10^{9} / \mathrm{L}$ while the median neutrophil value for other solid malignancies increased from $1.1 \times 10^{9} / \mathrm{L}$ to $3.0 \times 10^{9} / \mathrm{L}$. For patients with hematological malignancies, the median C-reactive protein (CRP) value increased from 4.5 to $5.0 \mathrm{mg} / \mathrm{L}$. For patients with breast cancer, the median CRP value increased from 5.2 to $5.6 \mathrm{mg} / \mathrm{L}$ and the median CRP value for patients with other solid tumors increased from 9.7 to $10.6 \mathrm{mg} / \mathrm{L}$.

\section{Infections}

Among patients for whom infection data were collected, $11.3 \%$ had an infection at first visit, $13.1 \%$ had an infection at the follow-up visit and $14.9 \%$ of the patients had an infection at a later chemotherapy cycle. Bacterial and viral infections were most frequently reported.

\section{Adverse Events}

In the course of the study, $29.8 \%$ of patients experienced $\geq 1 \mathrm{AE}$ that was at least potentially related to biosimilar filgrastim treatment (Table 9). The treatment related AEs reported most frequently were musculoskeletal and connective tissue disorders (12.3\%) with the preferred term (PT) bone pain (7.6\%); general 
Table 7 Chemotherapies, all-patients set

\begin{tabular}{|c|c|c|c|c|c|}
\hline Drug class & Substance & $\begin{array}{l}\text { Hematological } \\
\text { malignancies } \\
(N=48)\end{array}$ & $\begin{array}{l}\text { Breast cancer } \\
(N=233)\end{array}$ & $\begin{array}{l}\text { Other solid } \\
\text { tumors }(N=105)\end{array}$ & $\begin{array}{l}\text { Total } \\
(N=386)\end{array}$ \\
\hline \multirow[t]{7}{*}{ Antimetabolites } & Methotrexate & $1(2.1)$ & 0 & 0 & $1(0.3)$ \\
\hline & Gemcitabine & 0 & $4(1.7)$ & $5(4.8)$ & $9(2.3)$ \\
\hline & Fludarabin & $7(14.6)$ & 0 & $1(1.0)$ & $8(2.1)$ \\
\hline & Cytarabin & $1(2.1)$ & 0 & $1(1.0)$ & $2(0.5)$ \\
\hline & Capecitabin & 0 & $3(1.3)$ & $3(2.9)$ & $6(1.6)$ \\
\hline & Other & $1(2.1)$ & $1(0.4)$ & $2(1.9)$ & $4(1.0)$ \\
\hline & 5-Fluorouracil & 0 & $71(30.5)$ & $26(24.8)$ & $97(25.1)$ \\
\hline \multirow[t]{6}{*}{ Alkylating agents } & Oxaliplatin & $1(2.1)$ & 0 & $17(16.2)$ & $18(4.7)$ \\
\hline & Ifosfamide & 0 & 0 & $2(1.9)$ & $2(0.5)$ \\
\hline & Cyclophosphamide & $32(66.7)$ & $169(72.5)$ & $6(5.7)$ & $207(53.6)$ \\
\hline & Cisplatin & $1(2.1)$ & $3(1.3)$ & $17(16.2)$ & $21(5.4)$ \\
\hline & Carboplatin & 0 & $26(11.2)$ & $37(35.2)$ & $63(16.3)$ \\
\hline & Other & $10(20.8)$ & $1(0.4)$ & $1(1.0)$ & $12(3.1)$ \\
\hline \multirow[t]{7}{*}{ Intercalating agents } & Topotecan & 0 & 0 & $8(7.6)$ & $8(2.1)$ \\
\hline & Mitoxantron & 0 & $2(0.9)$ & 0 & $2(0.5)$ \\
\hline & Irinotecan & 0 & 0 & $9(8.6)$ & $9(2.3)$ \\
\hline & Etoposide & $4(8.3)$ & $1(0.4)$ & $18(17.1)$ & $23(6.0)$ \\
\hline & Epirubicin & 0 & $139(59.7)$ & $3(2.9)$ & $142(36.8)$ \\
\hline & Doxorubicin & $19(39.6)$ & $36(15.5)$ & $7(6.7)$ & $62(16.1)$ \\
\hline & Other & 0 & $1(0.4)$ & $2(1.9)$ & $3(0.8)$ \\
\hline \multirow[t]{5}{*}{ Mitotic inhibitors } & Vinorelbin & 0 & $7(3.0)$ & $2(1.9)$ & $9(2.3)$ \\
\hline & Vinflunin & 0 & 0 & $1(1.0)$ & $1(0.3)$ \\
\hline & Vincristine & $19(39.6)$ & 0 & $4(3.8)$ & $23(6.0)$ \\
\hline & Vinblastine & $2(4.2)$ & 0 & 0 & $2(0.5)$ \\
\hline & Other & $1(2.1)$ & 0 & 0 & $1(0.3)$ \\
\hline \multirow[t]{3}{*}{ Taxane } & Paclitaxel & 0 & $62(26.6)$ & $22(21.0)$ & $84(21.8)$ \\
\hline & Docetaxel & 0 & $88(37.8)$ & $7(6.7)$ & $95(24.6)$ \\
\hline & Other & 0 & $7(3.0)$ & $4(3.8)$ & $11(2.8)$ \\
\hline
\end{tabular}


Table 7 continued

\begin{tabular}{lllllr}
\hline Drug class & Substance & $\begin{array}{l}\text { Hematological } \\
\text { malignancies } \\
(\boldsymbol{N = 4 8 )}\end{array}$ & $\begin{array}{l}\text { Breast cancer } \\
(\boldsymbol{N}=\mathbf{2 3 3})\end{array}$ & $\begin{array}{l}\text { Other solid } \\
\text { tumors }(\boldsymbol{N}=\mathbf{1 0 5})\end{array}$ & $\begin{array}{l}\text { Total } \\
(\boldsymbol{N}=\mathbf{3 8 6})\end{array}$ \\
\hline Monoclonal antibodies & $\begin{array}{l}\text { Rituximab } \\
\text { Cetuximab }\end{array}$ & $28(58.3)$ & 0 & 0 & $28(7.3)$ \\
& Bevacizumab & 0 & 0 & $1(1.0)$ & $2(0.3)$ \\
& Other & $2(4.2)$ & $27(11.6)$ & 0 & $28(7.3)$ \\
Other cancer therapies & & $43(89.6)$ & $23(9.9)$ & $15(14.3)$ & $81(21.5)$ \\
\hline
\end{tabular}

disorders and administration site conditions (9.9\%) with the PTs mucosal inflammation (3.4\%) and fatigue (3.1\%); and blood and lymphatic system disorders $(9.2 \%)$ with the PTs neutropenia (6.5\%) and leukopenia (4.5\%). During the study, eight patients (2.1\%) reported serious adverse events (SAE) (Table 10) that were considered as potentially related to treatment of which one patient died with an SAE with insufficient information to define possible relationship to treatment.

Less than one percent $(0.8 \%)$ of patients had a delay in their chemotherapy due to FN after the first biosimilar filgrastim treatment and for $4.7 \%$ of patients, the chemotherapy in any subsequent cycle was delayed because of neutropenia. Reduction of the chemotherapy dose after first biosimilar filgrastim treatment due to $\mathrm{FN}$ was reported for $1 \%$ of patients and reduction of the chemotherapy dose in any subsequent cycle due to neutropenia was reported for $4.7 \%$ of patients.

\section{Patient-Reported Data}

The majority of patients administered the biosimilar filgrastim injection themselves at both treatment visits $(77.7 \%$ at first visit and $68.6 \%$ at last visit). On a scale of very difficult to very easy, most of the self-applicators reported that the application was easy or very easy. On a scale of 0 (no pain) to 10 (extreme pain) most patients perceived the pain of the biosimilar filgrastim injection as mild (median score 1.0).

\section{DISCUSSION}

$\mathrm{CIN}$ is a serious and potentially life-threatening consequence of cancer treatment mostly observed during the first cycles of chemotherapy [13-17]. Sepsis or severe infections are possible complications, however, delays and dose reductions of chemotherapy due to neutropenia in subsequent treatment cycles may be associated with compromised patient outcomes [18-22]. Although the prophylactic administration of antibiotics may reduce the incidence of $\mathrm{FN}$ and mortality related to infections [23], the guidelines of the European Organization for Research and Treatment of Cancer (EORTC) do not recommend this practice [24] due to possible emergence of antibiotic resistance. An alternative approach is the prophylactic treatment with G-CSF which decreases the incidence of $\mathrm{FN}$, the rate of hospitalization, and the use of antibiotics in patients at risk [25]. The guidelines of the American Society of 
Table 8 Leukocytes, neutrophils and C-reactive protein at visit 1, before the first and last chemotherapy cycles, stratified by type of tumour (safety-analysis set)

\begin{tabular}{|c|c|c|c|c|}
\hline \multirow[t]{2}{*}{ Visit $^{*}$} & \multirow{2}{*}{$\begin{array}{l}\text { Haematological } \\
\text { malignancies } \\
(N=47)\end{array}$} & \multicolumn{3}{|l|}{ Solid tumours } \\
\hline & & $\begin{array}{l}\text { Breast cancer } \\
(N=233)\end{array}$ & $\begin{array}{l}\text { Other } \\
(N=102)\end{array}$ & $\begin{array}{l}\text { Total } \\
(N=382)\end{array}$ \\
\hline \multicolumn{5}{|c|}{ Leukocytes $\left(10^{9} / \mathrm{L}\right)$} \\
\hline \multicolumn{5}{|c|}{ First visit } \\
\hline $\mathrm{N}$ & 43 & 219 & 95 & 357 \\
\hline Median & 3.2 & 4.3 & 2.9 & 3.6 \\
\hline \multicolumn{5}{|c|}{ Before first CT-cycle } \\
\hline $\mathrm{N}$ & 42 & 173 & 85 & 300 \\
\hline Median & 5.0 & 6.2 & 6.5 & 6.1 \\
\hline \multicolumn{5}{|c|}{ Before last CT-Cycle } \\
\hline $\mathrm{N}$ & 29 & 199 & 72 & 300 \\
\hline Median & 4.1 & 5.3 & 5.1 & 5.1 \\
\hline \multicolumn{5}{|c|}{ Neutrophils $\left(10^{9} / \mathrm{L}\right)$} \\
\hline \multicolumn{5}{|l|}{ First visit } \\
\hline $\mathrm{N}$ & 27 & 140 & 68 & 235 \\
\hline Median & 1.4 & 1.6 & 1.1 & 1.3 \\
\hline \multicolumn{5}{|c|}{ Before first CT-cycle } \\
\hline $\mathrm{N}$ & 27 & 117 & 55 & 199 \\
\hline Median & 2.8 & 3.7 & 3.9 & 3.7 \\
\hline \multicolumn{5}{|c|}{ Before last CT-cycle } \\
\hline $\mathrm{N}$ & 16 & 124 & 51 & 191 \\
\hline Median & 2.6 & 3.1 & 3.0 & 3.0 \\
\hline \multicolumn{5}{|c|}{ C-reactive protein $(\mathrm{mg} / \mathrm{l})$} \\
\hline \multicolumn{5}{|l|}{ First visit } \\
\hline $\mathrm{N}$ & 18 & 67 & 36 & 121 \\
\hline Median & 4.5 & 5.2 & 9.7 & 5.2 \\
\hline \multicolumn{5}{|c|}{ Before first CT-cycle } \\
\hline $\mathrm{N}$ & 15 & 34 & 27 & 76 \\
\hline Median & 5.3 & 5.1 & 12.9 & 6.8 \\
\hline \multicolumn{5}{|c|}{ Before last CT-cycle } \\
\hline $\mathrm{N}$ & 9 & 47 & 22 & 78 \\
\hline Median & 5.0 & 5.6 & 10.6 & 6.0 \\
\hline
\end{tabular}

* Lab values with comments were not included in the analyses

Oncology (ASCO) in 2006 and those of the National Comprehensive Cancer Network (NCCN) in 2014 recommend the use of G-CSF in primary prophylaxis for chemotherapy associated with a risk of $\mathrm{FN} \geq 20 \%$ [26]. The
2006 guidelines of EORTC updated in 2010 recommend the systematic use of G-CSF in primary prophylaxis to prevent $\mathrm{FN}$ if the risk of FN associated with the cytotoxic chemotherapy is $\geq 20 \%$, and in specific patients if the cytotoxic 
Table 9 AEs with at least potential relationship to study treatment

\begin{tabular}{ll}
\hline $\begin{array}{l}\text { System organ class (SOC)-preferred } \\
\text { term (PT) }\end{array}$ & $\begin{array}{l}\text { Total, } \\
\boldsymbol{N}=\mathbf{3 8 2}\end{array}$ \\
\hline Any & $114(29.8 \%)$ \\
Musculoskeletal and connective tissue & $47(12.3 \%)$ \\
$\quad$ disorders & $29(7.6 \%)$ \\
Bone pain & $38(9.9 \%)$ \\
General disorders and administration site & \\
$\quad$ conditions & $13(3.4 \%)$ \\
Mucosal inflammation & $12(3.1 \%)$ \\
Fatigue & $35(9.2 \%)$ \\
Blood and lymphatic system disorders & $25(6.5 \%)$ \\
Neutropenia & $17(4.5 \%)$ \\
Leukopenia & $27(7.1 \%)$ \\
Gastrointestinal disorders & $13(3.4 \%)$ \\
Nausea (3.4\%) &
\end{tabular}

A relationship was assumed unless 'no relationship' was recorded

Table 10 Serious adverse events with at least potential relationship to study treatment $(N=382)$

\begin{tabular}{ll}
\hline Preferred term (PT) & $\boldsymbol{n}(\%)$ \\
\hline Death & $1(0.3 \%)$ \\
Drug ineffective & $1(0.3 \%)$ \\
General physical health deterioration & $1(0.3 \%)$ \\
Pleural effusion & $1(0.3 \%)$ \\
Pulmonary embolism & $1(0.3 \%)$ \\
Neutropenia & $1(0.3 \%)$ \\
Atrial flutter & $1(0.3 \%)$ \\
Anal abscess & $1(0.3 \%)$ \\
\hline
\end{tabular}

A relationship was assumed unless 'no relationship' was recorded

chemotherapy induces a risk from 10 to $20 \%$ [24].

The use of biosimilar G-CSF (Nivestim) was approved by the European Medicines Agency
(EMA) for all the registered indications of the originator (Neupogen) including CIN, agranulocytosis and neutropenia due to infection with the HIV and mobilization of stem cells in the autologous and allogeneic settings, based on their comparable efficacy and safety profile to the originator G-CSF in CIN. Two phase I pharmacokinetic/ pharmacodynamic studies previously confirmed the similarity between biosimilar filgrastim and the reference product Neupogen $[27,28]$ and an extensive characterization study assessed the physiochemical similarity of biosimilar filgrastim to Neupogen [29]. Samples were analyzed for physicochemical properties, molecular characteristics, purity and biological activity. They were also compared after long-term storage to evaluate their degradation impurity profiles. Biosimilar filgrastim and Neupogen were shown to have comparable physicochemical properties, molecular characteristics, purity and biological activity.

A phase III double-blind study demonstrated the bioequivalence of biosimilar filgrastim and Neupogen in patients with breast cancer treated with doxorubicin and docetaxel in the neoadjuvant/adjuvant or first-line metastatic setting [30]. Among patients randomized to receive biosimilar filgrastim $(n=184)$ and Neupogen $(n=95)$, the mean duration of severe neutropenia in Cycle 1 was similar for biosimilar filgrastim (1.6 days; $n=165$ ) and Neupogen (1.3 days; $n=85$ ), meeting predefined criteria for bioequivalence. Secondary endpoints supporting bioequivalence included mean time to ANC recovery and incidence of FN. The biosimilarity of these agents was further demonstrated in a retrospective comparative cohort study of women with early breast cancer during (neo) adjuvant docetaxel/doxorubicin/ cyclophosphamide [31]. 
Through analysis of real-world day-to-day use, the results of the VENICE study extend the results of a recent review of available data, which concluded the safety of biosimilar filgrastim is similar to Neupogen [32]. In this study, the majority of the patients (64\%) received biosimilar filgrastim as primary prophylaxis and $36 \%$ as secondary prophylaxis. Because most of the patients were female with breast cancer, there was no widely used primary prophylaxis. Nevertheless, for some patients with breast cancer who received combination chemotherapy associated with a FN risk $>20 \%$ [i.e., TAC (docetaxel, doxorubicin, cyclophosphamide) Dose-dense AC/T (doxorubicin, cyclophosphamide, paclitaxel)] a primary prophylaxis with G-CSF is recommended [13]. Additionally, age is a risk factor [13] and justifies the prophylactic treatment of G-CSF. The median age was 61 and $39 \%$ of the patients were $>65$ years old.

The limitations of this study include that it is non-interventional, observational and lacks a blinded control. Additionally, a weakness is the lack of data captured, specifically regarding the chemotherapy regimens and severity of FN.

The use of biosimilar filgrastim according to the label in the VENICE observational study was effective and well-tolerated in both the primary and secondary prophylactic setting in patients undergoing CT for solid tumors and hematological malignancies. The rate neutropenia observed (Table 10) of $0.3 \%$ is lower than the rate of neutropenia in the baseline characteristics (2.6\%). Reduction of CT dose or delayed start of next CT cycle due to neutropenia were observed in approximately $1 \%$ of the patients after first biosimilar filgrastim treatment. No hospitalizations for FN occurred during the study; $2 \%$ of the patients were hospitalized because of infections. At least one
$\mathrm{AE}$ with at least potential relationship to treatment was reported by $30 \%$ of patients during the study. The most frequently reported AEs with at least potential relationship to treatment were in the SOC 'Musculoskeletal and connective tissue disorders' (12.3\%) with the PT bone pain (7.6\%).

Most patients administered the biosimilar filgrastim injection themselves at both treatment visits and the majority of the self-administrators reported that the application was easy or very easy. The perception of pain during the biosimilar filgrastim injection was mild for the majority of patients.

\section{CONCLUSION}

The VENICE observational study was designed to evaluate the use of Nivestim according to label-mandated posology for the administration of G-CSF and thereby provided additional real world data on supportive care for cancer patients. Biosimilar filgrastim was effective and well-tolerated in both the primary and secondary prophylactic settings in patients undergoing CT for solid tumours and haematological malignancies.

\section{ACKNOWLEDGMENTS}

Sponsorship, article processing charges, and the open access charge for this study were funded by Hospira Inc, A Pfizer Company, Lake Forest, IL, USA.

Editorial assistance in the preparation of this manuscript was provided by Dr. Anne Gentry and Dr. Carl Hornfeldt of Gentry Medical Communications Inc. This study was funded by Hospira Inc, which was acquired by Pfizer in 
September 2015. CR was an employee of Hospira and may own stock or options. All named authors meet the International Committee of Medical Journal Editors (ICMJE) criteria for authorship for this manuscript, take responsibility for the integrity of the work as a whole, and have given final approval to the version to be published. All authors had full access to all of the data in this study and take complete responsibility for the integrity of the data and accuracy of the data analysis. Presented as an Abstract at ECC-ESMO 2015 in Vienna, Austria. Available at: http://www. ejcancer.com/article/S0959-8049(15)30023-X/ abstract.

Disclosures. S. Fruehauf reports receiving lecture honoraria from Hospira (Prague, SIOG Meeting, 2015). B. Otremba reports that he is a consultant of Hospira/Pfizer Germany. O. Stötzer reports no conflict of interests. C. Rudolph was an employee of Hospira/Pfizer Germany at the time this manuscript was written.

Compliance with Ethics Guidelines. All procedures followed were in accordance with the ethical standards of the responsible committee on human experimentation (institutional and national) and with the Helsinki Declaration of 1964, as revised in 2013. Informed consent was not required from patients as this was a non-interventional observational trial.

Open Access. This article is distributed under the terms of the Creative Commons Attribution-NonCommercial 4.0 International License (http://creativecommons.org/licenses/ by-nc/4.0/), which permits any noncommercial use, distribution, and reproduction in any medium, provided you give appropriate credit to the original author(s) and the source, provide a link to the Creative Commons license, and indicate if changes were made.

\section{REFERENCES}

1. Villafuerte-Gutierrez P, Villalon L, Losa JE, Henriquez-Camacho C. Treatment of febrile neutropenia and prophylaxis in hematologic malignancies: a critical review and update. Adv Hematol. 2014;986938.

2. Fontanella C, Bolzonello S, Lederer B, Aprile G. Management of breast cancer patients with chemotherapy-induced neutropenia or febrile neutropenia. Breast Care (Basel). 2014;9:239-45.

3. Wang XJ, Lopez SE, Chan A. Economic burden of chemotherapy-induced febrile neutropenia in patients with lymphoma: a systematic review. Crit Rev Oncol Hematol. 2015;94:201-12.

4. Crawford J, Ozer H, Stoller R, et al. Reduction by granulocyte colony-stimulating factor of fever and neutropenia induced by chemotherapy in patients with small-cell lung cancer. $\mathrm{N}$ Engl J Med. 1991;325:164-70.

5. Trillet-Lenoir V, Green J, Manegold C, et al. Recombinant granulocyte colony stimulating factor reduces the infectious complications of cytotoxic chemotherapy. Eur J Cancer. 1993;29A:319-24.

6. Pettengell R, Gurney $\mathrm{H}$, Radford JA, et al. Granulocyte colony-stimulating factor to prevent dose-limiting neutropenia in non-Hodgkin's lymphoma: a randomized controlled trial. Blood. 1992;80:1430-6.

7. Ribeiro D, Veldwijk MR, Benner A, et al. Differences in functional activity and antigen expression of granulocytes primed in vivo with filgrastim, lenograstim, or pegfilgrastim. Transfusion. 2007;47(6):969-80.

8. Mhaskar R, Clark OA, Lyman $\mathrm{G}$, et al. Colony-stimulating factors for chemotherapy-induced febrile neutropenia. Cochrane Database Syst Rev. 2014;10:CD003039.

9. Nahon S, Mansour R, Meher B, Soumoudronga R, Gasnereau I, Labourey JL. Zarzio ${ }^{\circledR}$, biosimilar of filgrastim, in prophylaxis of chemotherapy-induced neutropenia in routine practice: a French prospective multicentric study. Support Care Cancer. 2015. doi:10.1007/s00520-015-2986-0. 
10. Gascon P. Presently available biosimilars in hematology-oncology: G-CSF. Target Oncol. 2012;7(Suppl 1):S29-34.

11. European Medicines Agency. Nivestim ${ }^{\mathrm{TM}} /$ filgrastim. EPAR Product Information. (05/01/2015 Nivestim -EMEA/H/C/001142 -N/0027). Available: http://www.ema.europa.eu/docs/en_GB/document library/EPAR_-_Product_Information/human/0011 42/WC500093661.pdf Date accessed 9/27/2016.

12. Kamioner D, Fruehauf S, Maloisel F, Cals L, Lepretre S, Berthou C. Study design: two long-term observational studies of the biosimilar filgrastim Nivestim $^{\mathrm{TM}}$ (Hospira filgrastim) in the treatment and prevention of chemotherapy-induced neutropenia. BMC Cancer. 2013;13:547.

13. Crawford J, Caserta C, Roila F, on behalf of the ESMO Guidelines Working Group. Clinical practice guidelines. Hematopoietic growth factors: ESMO Clinical Practice Guidelines for the applications. Ann Oncol 2010;21:(Supplement 5):248-251.

14. Crawford J, Dale DC, Kuderer NM, et al. Risk and timing of neutropenic events in adult cancer patients receiving chemotherapy: the results of a prospective nationwide study of oncology practice. J Natl Compr Canc Netw. 2008;6:109-18.

15. Timmer-Bonte JN, de Boo TM, Smit HJ, et al. Prevention of chemotherapy-induced febrile neutropenia by prophylactic antibiotics plus or minus granulocyte colony-stimulating factor in small cell lung cancer: a Dutch Randomized Phase III Study. J Clin Oncol. 2005;23:7974-84.

16. Vogel CL, Wojtukiewicz MZ, Carroll RR, et al. First and subsequent cycle use of pegfilgrastim prevents febrile neutropenia in patients with breast cancer: a multicenter, double-blind, placebo-controlled phase III study. J Clin Oncol. 2005;23:1178-84.

17. Wolff D, Culakova E, Poniewierski MS, Lyman GH, Dale DC, Crawford J. Predictors of chemotherapy-induced neutropenia and its complications: results from a prospective nationwide registry. J Support Oncol. 2005;3:24-5.

18. Cameron D. Management of chemotherapy-associated febrile neutropenia. Br J Cancer. 2009;101(Suppl 1):S18-22.

19. Hughes WT, Armstrong D, Bodey GP, et al. Support Care Cancer 2002 guidelines for the use of antimicrobial agents in neutropenic patients with cancer. Clin Infect Dis. 2002;34:730-51.

20. Krell D, Jones AL. Impact of effective prevention and management of febrile neutropenia. $\mathrm{Br} \mathrm{J}$ Cancer. 2009;101(Suppl 1):S23-6.
21. Padilla G, Ropka ME. Quality of life and chemotherapy induced neutropenia. Cancer Nurs 28:167-171 11 .

22. Wagner LI, Beaumont JL, Ding B, et al. Measuring health-related quality of life and neutropenia-specific concerns among older adults undergoing chemotherapy: validation of the Functional Assessment of Cancer Therapy-Neutropenia (FACT-N). Support Care Cancer. 2008;16(1):47-56.

23. Aapro MS, Cameron DA, Pettengell R, et al. EORTC guidelines for the use of granulocyte-colony stimulating factor to reduce the incidence of chemotherapy-induced febrile neutropenia in adult patients with lymphomas and solid tumours. Eur J Cancer. 2006;42:2433-53.

24. Aapro MS, Bohlius J, Cameron DA, et al. 2010 update of EORTC guidelines for the use of granulocyte-colony stimulating factor to reduce the incidence of chemotherapy-induced febrile neutropenia in adult patients with lymphoproliferative disorders and solid tumours. Eur J Cancer. 2011;47:8-32.

25. Lyman GH, Kuderer NM. Prevention and treatment of venous thromboembolism among patients with cancer: the American Society of Clinical Oncology Guidelines. Thromb Res. 2002;125(Suppl 2):S120-7.

26. Smith TJ, Khatcheressian J, Lyman GH, et al. 2006 update of recommendations for the use of white blood cell growth factors: an evidence-based clinical practice guideline. NCCN Clinical Practice Guidelines in Oncology. Myeloid Growth Factors. Version 2.2014. NCCN.org. J Clin Oncol. 2006;24:3187-205.

27. Waller CF, Bronchud M, Mair S, Challand R. Comparison of the pharmacodynamic profiles of a biosimilar filgrastim and Amgen filgrastim: results from a randomized, phase I trial. Ann Hematol. 2010;89:971-8.

28. Waller CF, Bronchud M, Mair S, Challand R. Pharmacokinetic profiles of a biosimilar filgrastim and Amgen filgrastim: results from a randomized, phase I trial. Ann Hematol. 2010;89:927-33.

29. Skrlin A, Radic I, Vuletic M, et al. Comparison of the physicochemical properties of a biosimilar filgrastim with those of reference filgrastim. Biologicals. 2010;38:557-66.

30. Waller CF, Semiglazov VF, Tjulandin S, Bentsion D, Chan S, Challand R. A phase III randomized equivalence study of biosimilar filgrastim versus Amgen filgrastim in patients receiving myelosuppressive chemotherapy for breast cancer. Onkologie. 2010;33:504-11. 
31. Brito M, Esteves S, André R, Isidoro M, Moreira A. Comparison of effectiveness of biosimilar filgrastim (Nivestim ${ }^{\mathrm{TM}}$ ), reference Amgen filgrastim and pegfilgrastim in febrile neutropenia primary prevention in breast cancer patients treated with neo(adjuvant) TAC: a non-interventional cohort study. Support Care Cancer. 2016;24(2):597-603.
32. Abraham I, Tharmarajah S, MacDonald K. Clinical safety of biosimilar recombinant human granulocyte colony-stimulating factors. Expert Opin Drug Saf. 2013;12:235-46. 